\title{
Criminologie
}

\section{Modèles vindicatoire et pénal en concurrence ? Réflexions à partir de l'expérience autochtone}

\section{Véronique Strimelle et Françoise Vanhamme}

Volume 42, numéro 2, automne-hiver 2009

Régulations sociopénales et peuples autochtones

URI : https://id.erudit.org/iderudit/038600ar

DOI : https://doi.org/10.7202/038600ar

Aller au sommaire du numéro

\section{Éditeur(s)}

Les Presses de l'Université de Montréal

ISSN

0316-0041 (imprimé)

1492-1367 (numérique)

Découvrir la revue

Citer cet article

Strimelle, V. \& Vanhamme, F. (2009). Modèles vindicatoire et pénal en concurrence ? Réflexions à partir de l'expérience autochtone. Criminologie, 42(2), 83-100. https://doi.org/10.7202/038600ar
Résumé de l'article

Qu'est-ce qu'un trouble, un conflit ? Comment les définit-on ? Comment y réagit-on ? Ces questions, qui composent la trame du présent article, s'insèrent dans nos travaux actuels qui explorent l'hypothèse de l'existence d'un modèle de justice actif dans nos rapports sociaux, mais caché par le discours dominant de la justice pénale : le modèle vindicatoire. Nous y discuterons plus particulièrement des questions soulevées par la rencontre entre les deux modèles de régulation, pénal et vindicatoire, et sur la façon dont ces deux modèles de régulation pourraient se côtoyer. Pour ce faire, nous nous intéresserons aux modes de régulation des troubles chez les Autochtones du Québec. Tout en mettant d'abord en lumière les principes de leur définition d'un trouble et de ses modes de régulation, nous essayerons d'en montrer la facette vindicatoire pour ensuite décrire comment s'est effectuée la rencontre des modèles de régulation autochtone avec le système de justice pénale. Nous dégageons enfin différentes pistes de réflexion quant à la survie et l'adaptation d'un tel modèle dans le contexte juridico-pénal occidental. 


\title{
Modèles vindicatoire et pénal en concurrence? Réflexions à partir de l'expérience autochtone
}

\author{
Véronique Strimelle \\ Professeure agrégée \\ Université d'Ottawa \\ vstrimel@uottawa.ca \\ Françoise Vanhamme \\ Professeure adjointe \\ Université d'Ottawa \\ fvanhamm@uottawa.ca
}

\begin{abstract}
RÉSUMÉ - Qu'est-ce qu'un trouble, un conflit? Comment les définit-on? Comment y réagit-on? Ces questions, qui composent la trame du présent article, s'insèrent dans nos travaux actuels qui explorent l'hypothèse de l'existence d'un modèle de justice actif dans nos rapports sociaux, mais caché par le discours dominant de la justice pénale: le modèle vindicatoire. Nous y discuterons plus particulièrement des questions soulevées par la rencontre entre les deux modèles de régulation, pénal et vindicatoire, et sur la façon dont ces deux modèles de régulation pourraient se côtoyer. Pour ce faire, nous nous intéresserons aux modes de régulation des troubles chez les Autochtones du Québec. Tout en mettant d'abord en lumière les principes de leur définition d'un trouble et de ses modes de régulation, nous essayerons d'en montrer la facette vindicatoire pour ensuite décrire comment s'est effectuée la rencontre des modèles de régulation autochtone avec le système de justice pénale. Nous dégageons enfin différentes pistes de réflexion quant à la survie et l'adaptation d'un tel modèle dans le contexte juridicopénal occidental.
\end{abstract}

MOTS-CLÉs - Autochtones, régulation des troubles et conflits, vindicatoire, pénal, pluralisme juridique.

\section{Introduction}

Toute forme organisée de vie collective se caractérise par un ensemble de valeurs, de normes, de règles, ainsi que d'organes de contrôle social formel et informel destinés à maintenir la cohésion et la vie du groupe 
(Paradelle, 2001; Delâge et Gilbert, 2003). De ce fait, l'apparition de troubles et leur gestion sont largement dépendantes des formes et règles en vigueur dans les différentes sociétés humaines. Ce concept de trouble, tel que nous l'entendons, englobe de façon plus large les situations qui dérangent ${ }^{1}$, soit des griefs humains, des frictions, des conflits, des événements qui rompent avec l'habituel, dont la pratique s'émeut car elles sont porteuses d'une norme ressentie. Elles sont de la sorte générées au sein de l'espace social qui est le «creuset des définitions des problèmes et des solutions» (Llewellyn et Hoebel, 1999: 27-28).

Et dans nos sociétés, c'est le système pénal, assureront certains, qui cimente notre vie collective en gérant la plupart des troubles et conflits qui y émergent. La présente contribution explore ces affirmations dans l'hypothèse d'une régulation vindicatoire des troubles et conflits. Mais d'abord, qu'entendons-nous par là? Pourquoi cette optique, et quel est son intérêt pour la criminologie? Ces éléments amènent progressivement à l'objet de la présente étude qui est de questionner la façon dont les deux modèles de régulation, pénal et vindicatoire, pourraient se côtoyer. En particulier, il s'agira de dégager les éléments qui rendent plausible cette coexistence tout en nous y rendant aveugles. Pour ce faire, notre exploration se fonde sur les modes de régulation des troubles chez les Autochtones du Québec. Tout en mettant d'abord en lumière les principes de leur définition d'un trouble et de ses modes de régulation, nous en montrerons la facette vindicatoire. Cette approche schématique permettra alors d'en explorer les points de rencontre avec le système de justice pénale et de dégager différentes pistes de réflexion quant à la survie et l'adaptation d'un tel modèle dans ce contexte juridico-pénal occidental. Pointer ces foyers de tension, c'est aussi contribuer à sortir du cadre juridico-pénal occidental, à mieux comprendre comment penser un mode de régulation sans se faire happer ou aveugler par son discours dominant.

\section{De la sanction pénale aux modes sociaux de régulation des troubles}

De nombreuses recherches visent à réfléchir à la sanction pénale et à l'introduction d'autres modes ou modalités de réaction à une situation

1. Le terme trouble évoqué ici se rapproche de la définition faite par Hulsman des «situations problèmes». Il les définit comme toute situation vécue ou perçue par un acteur quelconque comme posant problème, étant négative et inacceptable (Hulsman, 1982). 
problématique ou conflictuelle. Nos propres travaux, qui se situent également dans ce courant, se fondent sur la prémisse du recadrage systémique: l'on ne peut comprendre ce qui se passe à un niveau, ni donc tendre à le transformer, si l'analyse et l'action restent à ce niveau (Watzlawick et al., 1975). C'est pourquoi il nous semble fructueux de procéder à un recadrage de la sanction pénale, de la replacer dans le cadre plus large des modes sociaux de réaction à des troubles. Ce concept auquel nous faisons référence, le trouble, vise déjà à permettre de sortir du cadre des définitions juridiques puisque, comme nous l'avons déjà mentionné, il désigne plus largement les situations qui dérangent. Cette optique de recadrage se trouve d'emblée confortée par le fait que, suivant différents résultats de recherche, le pénal ne se charge que d'une petite part des troubles. Certains peuvent en effet entrer dans le domaine légal, civil, administratif (cf. les illégalismes privilégiés, Acosta, 1987) et d'autres échappent totalement à toute définition légale. Et ensuite, la gestion de ces troubles n'est pas l'apanage de la seule réaction pénale, même quand ces situations tombent ou pourraient tomber sous le coup de la loi. Certaines peuvent être traitées par d'autres formes de règlement (civil, administratif) ou échapper à toute forme de prise en charge formelle (Robert, 1974; 1977 ; Acosta, 1987). Diverses recherches basées sur des enquêtes de délinquance autorévélée ou des sondages de victimisation ont de plus souligné à quel point le pénal ne traitait que peu de cas criminalisables (Cousineau et Cucumel, 1991 : 57-80; Commission du droit du Canada, 2003 : iii-24; Junger Tas et al., 2003 ).

C'est ainsi qu'émerge une question qui nous semble fondamentale, celle de savoir ce que font les gens pour réguler les situations de trouble qu'ils rencontrent. Dans la foulée, sortant ici aussi de la définition pénale et à l'instar d'Hulsman, la question se pose de savoir ce qu'ils considèrent comme trouble: comment reconnaît-on une situation «qui ne va pas», une situation qui appelle à sa régulation, à une réaction? Ces questions sont d'autant plus intéressantes que la vie collective se poursuit, se transforme et se maintient dans tous les conflits qui la traversent, qu'elle «tient» globalement, sans donc une intervention pénale généralisée. Qu'est-ce qui permet à la société de tenir ainsi? Remarquons que cette interrogation présuppose que les modes sociaux de régulation des troubles, dans la vie quotidienne, ne sont pas de simples réactions conjoncturelles purement contingentes dont il n'y aurait rien à dire. Au contraire, l'on serait en présence d'une institution 
sociale, c'est-à-dire un fait social sédimenté dans l'histoire et donc caractérisé par un degré élevé de permanence, et qui oriente les pratiques (Javeau, 1994).

À ce sujet, un modèle coutumier de régulation des troubles a été exploré dans une étude multidisciplinaire dirigée par Verdier, le modèle vindicatoire (Verdier et al., 1980; 1984). Il s'agit d'un système de contrôle social actif dans des communautés traditionnelles, qui régule les échanges et médiatise la violence qui pourrait en émerger (Verdier, 1980: 16; Hénaff, 2000). La réaction peut prendre une variété de formes suivant la culture dans laquelle elle s'enracine, mais une même logique de fond les anime (Hénaff, 2000). Dans la perspective vindicatoire traditionnelle en effet, lorsqu'une personne a été offensée, son clan, en tant que groupe social, se sent impliqué dans son honneur et sa puissance, dans sa capacité d'interagir avec les autres groupes suivant un statut d'égalité, d'équivalence. L'offensé et son groupe deviennent par là même les créanciers d'une atteinte équivalente. Comme le groupe n'a ni obligation ni intérêt à la mise en œuvre de cette créance, l'offenseur ou son clan sont appelés à réparer, c'est-à-dire à rendre son statut d'adversaire, d'égal au groupe atteint. Le principe, c'est d'assurer la continuité des relations, la re-solidarisation et donc l'inclusion dans l'espace social auquel les deux clans adhèrent (Verdier, 1980)2 ${ }^{2}$ La relation vindicatoire favorise ainsi la recherche d'une solution permettant aux intéressés de retrouver leur statut d'équivalents (équilibre des relations), et au groupe de préserver son intégrité et sa survie en évitant un déferlement de vendettas (gestion de la violence), de «tenir» (Alline, 2004: 8). La relation entre personnes ou groupes en conflit y est donc une relation d'adversité liant des partenaires qui se reconnaissent à la fois comme identiques et différents, mais non comme des ennemis (Hénaff, 2000: 50-54). Des solutions radicales d'exclusion, comme le bannissement ou la mort, ne sont envisagées que dans les cas extrêmes, en dernier recours.

Les travaux de Verdier soutiennent que ce système aurait persisté sous une forme principalement dénaturée dans les États modernes, cédant la place à la justice étatique (Verdier, 1980: 36). Dans le processus de centralisation et de constitution de l'État-nation en effet, l'État

2. Dans le système global de réaction, la contrepartie de l'obligation vindicatoire en réponse à un dommage produit par un individu extérieur au clan, c'est la sanction expiatrice en réaction à une transgression par un des siens. Dans de tels cas, les rituels collectifs visent ici aussi à restaurer l'unité et l'intégrité du groupe et donc, au but du compte, à réintégrer le transgresseur (Verdier, 1980: 22-23). 
a monopolisé la violence légitime, la gestion des échanges sociaux et de la justice. L'individu, lui, y a perdu une partie du pouvoir dont il disposait dans ses rapports sociaux concrets (Courtois, 1984): le système pénal a confisqué la place de la victime (Foucault, 1975) et se faire justice soi-même est devenu une atteinte à la légitimité étatique (Verdier, 1980; Verdier, 2004). En chemin, le système vindicatoire a été non seulement banni, mais aussi diabolisé (Verdier, 1980; Alline, 2001):

Il y a un discrédit très fort de la vengeance dans notre société moderne, qui tient à son refoulement et à sa dénaturation par l'État: avec l'étatisation de la justice, la vengeance est devenue une justice privée, dé-socialisée et dé-ritualisée; vengeance sauvage, que les historiens du droit pénal ont utilisée comme "notion-repoussoir» pour mieux asseoir la rationalité de la peine. (Verdier, 1984: 150)

C'est dans la foulée de ces réflexions que nous développons actuellement un travail empirique et théorique sur les pratiques actuelles de régulation sociale en nous appuyant, à titre heuristique, sur ce modèle vindicatoire qui se serait toutefois adapté aux caractéristiques d'une société étatisée, centralisée et organique. À cet égard, l'hypothèse posée est que, malgré sa dimension présumée d'institution sociale, il ne se retrouverait que dans les pratiques, au niveau implicite et seen but unnoticed de l'action, du fait de la dominance de la justice pénale, dont la force épistémique nous aurait de plus rendus aveugles à cette forme de réaction. C'est cette hypothèse que nous voulons alimenter ici par l'examen d'une situation connue de la recherche, où les deux modèles de régulation, pénal et vindicatoire, se côtoient. Comment se rencontrent-ils? Quels sont les concepts, les rationalités où les modèles s'entrechoquent, entrent en concurrence, s'emmêlent, où l'un cède le pas à la logique de l'autre, se fait happer ou bien y résiste, ouvertement ou non?

Pour répondre à ces questions, la référence aux peuples autochtones ${ }^{3}$ du Québec nous a paru fructueuse. Depuis les années 1970, la recherche

3. Bien que le concept d'autochtone fasse l'objet de débats (Simard, 2003; Dorais, 2009; Martin, 2009), nous utiliserons ce terme pour désigner les peuples descendants des premiers occupants qui, au moment de la conquête au xvi siècle par les colons, vivaient sur le territoire du futur Québec. Actuellement, onze nations autochtones sont officiellement reconnues sur le territoire québécois. Parmi elles, dix sont des nations amérindiennes et la onzième est formée des Inuits du Nunavik (Jaccoud, 1995; Paradelle, 2001; Lajoie, 2008; Martin, 2009). Au Québec, programmes et réseaux de recherche sur les Autochtones sont actifs au sein des universités, des centres de recherche. Citons notamment le réseau de recherche et de connaissance DIALOG créé à l'Institut national de la recherche scientifique (Lévesque, 2009: 457); le programme de recherche «Peuples autochtones et 
s'y est de plus en plus intéressée, en lien avec la montée des revendications liées à leur autonomie politique (Jaccoud, 1999; Lévesque, 2009). La mise sur pied de la Commission royale sur les peuples autochtones en 1991 a encore accentué le mouvement en favorisant la publication de nombreux travaux portant sur l'histoire, la culture, le savoir, les sociétés autochtones (Lévesque, 2009: 460), et notamment sur la question de leur «justice» (Canada, 1991; Hamilton et Sinclair, 1991; Canada, 1993 ; Canada, 2006), sur leur approche spécifique des troubles et conflits qu'en dépit des effets dévastateurs de la colonisation, certaines communautés autochtones semblent avoir réussi à garder ou à restaurer.

Quelques précisions s'imposent quant à la méthodologie suivie ici. D'abord, nos sources sont écrites. À cet égard, il faut souligner que nombre de rapports de recherche ont été produits par des chercheurs non autochtones dont l'interprétation, les présupposés influencés par notre culture juridique occidentale ont pu teinter l'appréhension des questions et des valeurs autochtones (Rouland, 1984; Paradelle, 2001; Lévesque, 2009; Martin, 2009). Dans la mesure du possible, nous avons retenu les travaux tenant compte justement de ces biais culturels. Ensuite, nous nous sommes basées sur la documentation disponible portant sur les modes de règlement des troubles propres aux Autochtones et décrits par des chercheurs ayant travaillé sur le terrain auprès de certaines communautés, notamment de la communauté inuite (Rouland, 1983 ; Dorais, 1984; Jaccoud, 1995 ; Paradelle, 2001, Lacasse, 2004; Jaccoud et Johnson, 2007). Enfin, précisons qu'il n'existe certes pas un modèle autochtone universel de réponse aux troubles. Les communautés autochtones se caractérisent par des modes de vie, des environnements fort contrastés. Leurs rapports avec les occupants blancs ont aussi varié en intensité. Leurs définitions et attitudes par rapport aux transgressions des normes diffèrent donc nécessairement (Lajoie, 2008; Martin, 2009). Cependant, on peut dégager certains traits plus ou moins communs à leurs modes de réaction qui apportent suffisamment d'éléments d'information pour la présente exploration. C'est de ce noyau dont nous traiterons, en admettant bien sûr que cela ne rend pas

gouvernance», financé par le Conseil de recherche en sciences humaines du Canada (CRSH), qui vise à déterminer des modèles de gouvernance autochtone et regroupe 35 chercheurs issus de différentes disciplines universitaires (Lajoie [Ed.], 2007); la chaire de recherche du Canada sur la condition autochtone comparée à l'Université Laval. 
toute la complexité, la disparité et les nuances des divers modes existant pour chaque communauté, ni d'ailleurs pour chaque individu ressortissant actuellement d'un groupe autochtone. Ces traits que nous schématisons font ainsi référence à la coutume typique, telle qu'elle a toutefois été rapportée par les recherches empiriques contemporaines ${ }^{4}$.

\section{Modes autochtones de régulation des troubles: survivance et traces vindicatoires}

Les recherches menées auprès des communautés autochtones québécoises soulignent à quel point le système juridique euro-américain imposé à la suite de la colonisation a pu coexister avec leurs propres modes de régulation (Delâge et Gilbert, 2002; Lajoie, 2008; Martin, 2009). Dans les années 1980, une étude sur l'acculturation judiciaire des Inuits du Canada soulignait ainsi que ces derniers cherchaient toujours en premier lieu à régler eux-mêmes leurs problèmes dans le cadre familial ou communautaire (Rouland, 1983: 184). Mais la relation est évidemment déséquilibrée, le système euro-américain prédomine. Il impose non seulement sa loi, mais aussi sa logique d'action. La même recherche précisait en effet que les Inuits, dans les milieux semi-urbains en particulier, faisaient de plus en plus appel aux institutions étatiques pour régler leurs problèmes (Rouland, 1983: 184). L'imposition du modèle de justice euro-américain a dans bien des cas entraîné la perte des anciens mécanismes de règlement des différends, favorisant une dépendance accrue des Autochtones par rapport aux institutions de l'État considérées désormais comme seules aptes à régler leurs problèmes (Canada, 1993 ; Jaccoud, 1999; Canada, 2006). Parmi les autres effets dévastateurs de ces politiques colonisatrices, citons aussi la perte des valeurs, de l'identité culturelle et du lien social, mais aussi la pauvreté, l'alcoolisme, les abus physiques, ou encore le suicide...

Malgré ce recul devant le système pénal, un mode autochtone de résolution des conflits subsistait néanmoins; depuis, il est même revendiqué. En effet, la montée des mouvements en faveur de l'autonomie politique des peuples autochtones a fait ressortir les revendications de ces peuples portant notamment sur la mise en place de formes de justice

4. Cela n'implique donc pas que ces valeurs et pratiques font l'unanimité parmi les peuples autochtones ni qu'elles représentent une sorte d'idéal de sagesse et d'harmonie, exempt de tout défaut et dysfonctionnement (Lajoie, 2008; Martin, 2009). 
plus adaptées à leurs besoins et à leur culture (Jaccoud, 1999; Lévesque, 2009). Ces revendications coïncidaient aussi avec la volonté des autorités gouvernementales de rendre à ces collectivités spoliées la place qu'elles exigeaient au sein de la confédération canadienne, en tenant compte de leurs spécificités, mais aussi en reconnaissant les effets qu'ont exercés et qu'exercent encore aujourd'hui sur ces peuples plus de cinq cents ans de colonisation européenne. Des politiques et pratiques pénales jugées plus adaptées aux valeurs autochtones ont alors été créées en vue de répondre à ces demandes (Jaccoud et Johnson, 2007: 207). Et bien que les interviewés de diverses communautés autochtones du Québec considèrent que ces nouvelles structures ne leur accordent qu'un pouvoir consultatif, aux yeux de certains, elles constituent une opportunité pour reprendre ultérieurement le contrôle de leurs modes de régulation dans leur communauté: soit en collaborant avec l'appareil pénal étatique, soit en transférant les structures et les ressources aux communautés, sans référence au cadre étatique (Jaccoud et Johnson, 2007: 208). Le mode autochtone de régulation des troubles est par conséquent d'autant plus vivant qu'il est revendiqué, rendu visible et reconnu, du moins sous certaines formes. Et l'on peut supposer que c'est dans le champ des échanges quotidiens et informels qu'il garderait une plus grande vitalité puisque ce champ est moins investi par les appareils étatiques de contrôle ${ }^{5}$. En effet, «rendre justice» n'est pas seulement une question d'institutions, la notion déborde largement du judiciaire: elle renvoie plus largement au sentiment $\mathrm{du}$ «juste» comme échange social (Courtois, 1984: 24; Kellerhals et al., 1988; Garnot, 2000). Qu'en est-il chez les Autochtones?

La vision du monde qui se dégage de leurs valeurs est holistique et fondamentalement imprégnée par la notion de cercle. Ce cercle symbolise l'idée de totalité, de perfection, d'équilibre, d'égalité et d'harmonie entre toutes les formes de vie, il représente l'univers. Celui-ci se présente ainsi comme une grande chaîne de relations où toute personne est à la fois équivalente aux autres et dépendante d'eux (Dumont, 1993; Sioui, 1999; Jaccoud, 1999; Paradelle, 2001). Cette interdépendance pousse alors à pratiquer le partage et la collaboration entre membres du groupe. Dans cette perspective, il est difficile de distinguer l'intérêt

5. Précisons que cette question appelle à un travail de terrain et à l'observation longue et assidue des pratiques usuelles du quotidien, et qu'elle n'a pas été traitée en tant que telle à notre connaissance. 
personnel de celui du groupe, de distinguer le «soi» des autres (Dumont, 1993; Paradelle, 2001).

En conséquence, la coutume amérindienne n'évalue en général pas les situations comme bonnes ou mauvaises, mais comme porteuses ou non d'harmonie (Ross, 1996: 123). Les communautés reconnaissent de ce fait que les troubles font partie de la vie, tout en préconisant cependant qu'ils doivent être réglés afin de préserver l'harmonie des relations, garante de la survie du groupe (Dorais, 1984; Sioui, 1999; Paradelle, 2001). Traitant du concept d'offense dans la langue des Inuits du Labrador septentrional, Dorais (1984) souligne ainsi que la connotation de base de ce concept ne renvoie à aucun contenu moral, mais désigne plutôt le manque de mesure, d'ajustement. Le trouble se révèle alors être quelque chose qui agace, qui provoque l'inconfort, qui est incorrect (Dorais, 1984: 6). La dimension collective en apparait lorsque ces Inuits considèrent cette offense comme une humiliation faite à «ceux et celles qui en sont les victimes, mettant ainsi en danger l'harmonie sociale du groupe» (Dorais, 1984: 7), pouvant en affecter l'énergie vitale. Un trouble peut alors être défini comme ce qui altère ou perturbe l'idéal d'harmonie, le cercle. Et c'est l'impact sur la communauté qui détermine son existence et son registre de gravité.

La logique de sa régulation, c'est alors de restaurer l'intégrité perdue: intervient ici le concept de guérison, central dans les traditions autochtones. Ce concept signifie, sur un plan général, le retour à un sentiment d'équilibre et de globalité (Jaccoud, 1999). Transmettre les valeurs de la communauté et veiller à leur respect relève traditionnellement de la responsabilité de la parenté au sens large. Ce sont en effet pour l'essentiel les familles qui règlent les différends au quotidien (MontureOkanee, 1993). On voit ici que la conception autochtone de ce qui est acceptable repose sur l'apprentissage, l'intériorisation et la mise en pratique des valeurs au quotidien, c'est-à-dire la socialisation. Et le ciment qui «tient» la société, qui permet le maintien de l'équilibre social, repose donc sur la force des coutumes et traditions, qui sont transmises par les aînés et les sages (Dumont, 1993).

$\mathrm{Du}$ fait de leur faible impact sur la vie communautaire, certains comportements dérangeants peuvent n'entraîner qu'une régulation mineure. On va par exemple recourir aux commérages, à la moquerie ou aux sobriquets. Par contre, quand une situation est estimée plus menaçante pour la survie du groupe, les modes de régulation mobilisent et impliquent la communauté dans son ensemble (Paradelle, 2001). 
Selon la coutume, chaque membre du groupe a en effet le droit d'intervenir et de participer au même titre que les autres. La décision finale est le produit du consensus. Elle est considérée comme un moyen de redonner à chacun sa place et de rétablir les relations dégradées (Dumont, 1993). Dans les processus traditionnels, les sanctions comportaient généralement une réparation publique et non sanglante pour éviter le recours à une perte de vies humaines, même si le bannissement ou l'exécution pouvaient advenir (Sioui, 1999; Jaccoud, 1999; Paradelle, 2001). Cette réparation pouvait consister en services, en présents offerts dans un cadre rituel, ceux-ci étant alors perçus comme offres de paix afin d'ôter tout désir de vengeance ou en excuses (Sioui, 1999). Enfin, le processus de guérison pouvait se finaliser lors de cérémonies de purification (Monture-Okanee, 1993).

Quoique fort brève, cette schématisation des modes autochtones de régulation des troubles laisse transparaître différents indices au principe du modèle vindicatoire ${ }^{6}$. L'on a en effet constaté que ces modes sont organisés en vue de préserver l'intégrité du groupe et de gérer la violence immodérée susceptible d'émerger des différends. Ce primat de l'intérêt du groupe tient aux conditions dans lesquelles évoluent la plupart des sociétés traditionnelles, dont les Autochtones. Face à un environnement hostile et à la dureté des conditions matérielles, ces communautés doivent s'organiser de manière à conserver leurs forces vives et ainsi éviter leur disparition à court ou à long terme (Rouland, 1983 ; Paradelle, 2001). La notion d'équivalence de statut est aussi prépondérante: égalité entre personnes qui réagissent en tant qu'offenseur ou offensé, égalité affectée par l'acte perturbateur et par la dette à

6. On peut à juste titre faire aussi le lien entre les modèles de justice restauratrice et la réaction dans une socialité vindicatoire. En effet, dans les modèles restauratifs, l'objectif opérationnel est de permettre aux communautés et aux individus de se réapproprier leurs conflits à travers des modes consensuels de gestion de ceux-ci, par la négociation. Cela permet à la personne mise en cause de réparer le tort commis, de favoriser sa réintégration dans la société, ce qui correspond à une restauration de l'équivalence de statuts. L'objectif général est ainsi le retour à un équilibre affecté par l'acte problématique, la reconstitution du lien social (Bonafé-Schmitt, 2003 : 37). Une proximité entre les deux approches existe, mais on ne peut pourtant confondre l'une avec l'autre. En effet, l'origine de la démarche restauratrice reste, dans l'état des choses, largement définie par rapport au droit et à la pénalité, son point d'ancrage diffère donc grandement de celui à partir duquel se développe la socialité vindicatoire. À cet égard, il semble intéressant de se demander si les principes $\mathrm{du}$ modèle vindicatoire pourraient constituer une toile de fond théorique apte à articuler les différentes versions de la justice restauratrice et affranchir celle-ci de la rationalité juridico-pénale moderne (Pires, 2001; Lalonde, 2003; Noreau, 2003; Strimelle, 2007). 
honorer, égalité rétablie par le processus de guérison qui rend au groupe sa cohésion et aux parties leur position. Les modes de réparation sont destinés non à compenser la valeur matérielle de la perte subie, mais bien celle de la créance de vengeance, s'adressant au statut symbolique de la personne ou du groupe offensé. Le recours à la vengeance au sens vindicatoire favorise de la sorte un système d'échanges contrôlé qui obéit «à une recherche de parité et d'équilibre» (Courtois, 1984: 24). En ce sens, telle que nous la trouvons dans les pratiques coutumières autochtones, elle fonctionne comme une institution sociale car toujours comprise à l'intérieur des normes et règles produites par le groupe, sédimentée dans son histoire (Verdier, 1980; Courtois, 1984).

\section{Réflexions conclusives. Modèles vindicatoire et pénal: coexistence et résistances}

Comme le soulignent Rouland (1983), Dorais (1984) et Paradelle (2001) au sujet des Inuits, il est difficile de traiter de notions comme le crime, le droit, la culpabilité et la peine dans des cultures ${ }^{7}$ qui ne comptent ni ces concepts dans leur registre, ni ces mots dans leur vocabulaire. De telles conceptions n'y ont aucun sens car elles sont totalement absentes de leur organisation sociale et de leurs représentations de la place de l'humain dans le monde sacré et profane. Il y a une situation qui est évaluée inappropriée pour l'équilibre du groupe, en fonction des circonstances: on est loin de l'acte défini a priori comme mauvais, de la notion codifiée du crime. Le cercle, qui symbolise cet idéal d'harmonie, est mobilisé au quotidien en tant que référence normative à laquelle il faut tendre. Dès lors, dans la vision du monde développée par les sociétés autochtones, le conflit, les différends font partie intégrante du quotidien. Implicitement, la société est par conséquent interprétée dans une perspective conflictuelle tendant vers cet idéal d'harmonie, ce qui diffère totalement d'un droit qui se dit fondé sur le consensus social. La question de la culpabilité ne se pose pas dans les termes euro-américains: c'est davantage celle des conséquences relationnelles globales que celle du mens rea, de l'intention «criminelle»

7. Le terme «culture» est à comprendre ici comme un ensemble de traditions, de règles, de symboles qui influencent les manières d'être, de penser, de faire, de ressentir d'un groupe de personnes: " "Culture". Symbolic vebicles of meaning, including beliefs, ritual practices, art forms, ceremonies, as well as informal... practices such as language, gossip, stories and rituals of daily life» (Swidler, 1986: 273). 
(maligne?). Par ailleurs, l'égalité entre les membres de ces sociétés commande le respect pour chacun et suppose l'existence de relations qui ne sont pas fondées sur le pouvoir, sur l'autorité d'un chef ou de son représentant (Clastres, 1974 ; Dumont, 1993 ; Sioui, 1999; Paradelle, 2001); la décision y est consensuelle. Celle-ci n'est en général pas prise à partir des notions de punition et de rejet (bien que l'exclusion existe), mais bien de celle de rétablissement des statuts, relations et équilibre du groupe. Les réparations elles-mêmes ne portent pas sur le même objet: apaisement du côté autochtone, valeur matérielle perdue de l'autre. Quant à la guérison, c'est un processus holistique visant à favoriser le retour à un équilibre personnel et communautaire. Elle concerne tous les aspects de l'être - physique, psychologique, affectif et spirituel - et ne revêt donc pas un sens individualiste et thérapeutique. De ce fait, elle s'effectue au moyen de relations et non dans l'isolement (Jaccoud, 1999).

Bref, l'examen des pratiques autochtones nous apprend combien la logique juridique euro-américaine est fondamentalement étrangère à leurs valeurs et combien il est erroné de se fonder sur sa notion de «justice» dans un modèle vindicatoire. D'ailleurs, plusieurs langues des Premières Nations ne disposent d'aucun terme pour désigner ce concept :

Lorsque je me suis renseignée pour la première fois sur la traduction du mot justice en ojibwa, on m'a dit que ça se disait «ti-baq-nee-qwa-win». Littéralement, cela signifie «système pour quelque chose qui a déjà mal tourné». Le système en question, c'est le système judiciaire euro-canadien. $[\ldots] \mathrm{Au}$ cours de notre conversation, la grand-mère à qui je parlais m'a répété plusieurs fois que le terme justice n'existait pas dans la langue ojibwa. (Monture-Okanee, 1993: 121-122)

Une telle différence de rationalité a pourtant permis aux modes autochtones de régulation des troubles de subsister. Certes, les revendications sociopolitiques de ces communautés ont contribué à ce maintien, tout en soulevant des questions sur la pertinence et l'authenticité de certaines formes réactualisées de «justice» autochtone comme les cercles de sentence (Jaccoud, 1999). Mais il convient de souligner aussi que l'exercice d'une approche vindicatoire ne s'applique pas seulement dans le cadre de circonstances exceptionnelles ou jugées graves, elle se noue aussi dans les contacts quotidiens, familiaux... en fait partout où les conflits émergent et se règlent. En tant que fait social, il est impossible de parler de vindicatoire public ou privé, car le rapport entre partenaires dans une 
famille comme dans la communauté est toujours médiatisé par des règles partagées, un code idéologique où le sentiment du «juste» comme échange est fondé dans la socialisation. Il transcende donc les institutions et les catégories imposées par le système de justice euro-américain (Courtois, 1984: 24; Garnot, 2000). La vie quotidienne fournit ainsi un large ancrage à la survivance de pratiques vindicatoires.

Les modes par lesquels le lien social rend possible la vie collective s'opèrent au quotidien sous des formes multiples, créatives, variées et informelles, mais néanmoins porteuses de sens. Les valeurs qui fixent ce qui est désirable s'organisent de manière à (re)produire une certaine vision du monde et influencent la manière d'interpréter la réalité. Elles se reflètent dans le comportement envers les autres et le milieu naturel (Pollner, 1991: 83 ; Dumont, 1993 ; Boudon et al., 1999: 243). Ainsi, selon l'exemple donné par les Autochtones, la définition du trouble et ses modes de régulation au quotidien sont interconnectés. Ils reposent sur leur vision de la vie collective où l'individu est membre de plein droit, où sa «reconnaissance» personnelle et relationnelle est fondamentale et présupposent et imposent l'exigence de réciprocité (Kellerhals et al., 1997: 126; Honneth, 2000). Il y a là une relation étroite de sens apte à produire non seulement du lien social, mais aussi les conditions de légitimation sociale, et donc de perpétuation des pratiques vindicatoires. Précisons qu'il reste en suspens la question de l'effet de la notion centrale d'intégrité du groupe lorsque sa survie n'est pas matériellement menacée.

Finalement, l'hypothèse que ce modèle, adapté aux caractéristiques d'une société étatisée, centralisée et organique, se retrouverait dans la pratique au niveau implicite de l'action s'en trouve confortée. Nombreux sont les codes acquis par la socialisation qui ne s'actualisent qu'en situation concrète, qui ne font pas l'objet de conversations et qui ne sont pas théorisés, qui sont simplement vécus. Les propriétés du social peuvent en effet être vues, mais non remarquées (seen but unnoticed) (Garfinkel, 1997 : 35-36). La force de l'activité pratique et des concepts pénaux n'est pas la seule en cause. Ils constituent plutôt un voile supplémentaire qui contribue à occulter l'idée même de la potentialité d'une forme de réaction vindicatoire dans notre vie collective occidentale. C'est la dimension de «l'impensé du pluralisme» que nous visons ici. En effet, dans nos sociétés à héritage judéo-chrétien, le concept unitaire du pouvoir (divin ou séculier) a induit que l'on ne peut y concevoir l'existence que d'un seul droit; la potentialité de la dualité ou de la pluralité «juridique» s'en 
trouve exclue. Ce qui constitue une différence de plus avec des cultures traditionnelles comme celles des Autochtones, où la régulation ne vient pas d'une force extérieure (comme dieu ou le souverain de droit divin) «mais de l'intérieur du groupe dans lequel, par un effort de domestication de ses énergies (ou de sa violence)», tout par contre y «est pensé en termes multiples [...] et interdépendants» (Le Roy, 1999: 76-78). D'autres recherches et analyses ont souligné à ce propos la capacité d'absorption de la rationalité juridico-pénale, qui s'approprie le discours réparateur, mais le dénature et le transforme d'après sa propre grille d'interprétation (Lalonde, 2003 ; Strimelle, 2007).

À cet égard, et pour terminer, il ne nous semble pas inutile de souligner qu'en examinant les différents écrits relatifs à la question traitée dans la présente contribution, nous avons remarqué l'usage fréquent de termes tels que les droits traditionnels, les traditions juridiques, les droits ancestraux pour traiter des modes autochtones de gestion des troubles. Même s'ils font référence à une acception anthropologique du droit, ces termes risquent toujours d'induire une certaine vision de la régulation des troubles autochtone, calquée sur le modèle occidental de «justice», en sous-entendant par exemple l'existence du juridique, de l'infra-juridique et de l'extra-juridique dans ces sociétés. Traiter des traditions juridiques exclut alors d'office toutes les situations qui sont considérées comme ressortissant du règlement privé des conflits interindividuels, quand celles-ci ne font pas l'objet d'une intervention visible ou reconnue.

\section{Références}

Acosta, F. (1987). À propos des illégalismes privilégiés. Réflexions conceptuelles et mise en contexte. Criminologie, 21 (1), 7-34.

Alline, J.-P. (2001). Les victimes: des oubliées de l'histoire du droit? In Association française pour l'Histoire de la Justice (Ed.), La cour d'assises. Bilan d'un héritage démocratique (247-268). La Doc. Française, Coll. Histoire de la justice, 13.

Alline, J.-P. (2004). Gouverner le crime: les politiques criminelles françaises de la révolution au XXI siècle. Paris: L'Harmattan.

Bonafé-Schmitt J.-P. (2003). Justice réparatrice et médiation pénale: vers de nouveaux modèles de régulation sociale? In M. Jaccoud (Ed.), Justice réparatrice et médiation pénale: convergences ou divergences? (17-49). Paris: L'Harmattan.

Boudon, R., Besnard, P., Cherkaoui, M., \& Lécuyer, B.-P. (1999). Dictionnaire de sociologie. Paris: Larousse-Bordas. 
Canada (1991). Les peuples autocbtones et la justice pénale. Ottawa: Commission de réforme du droit.

Canada (1993). Les peuples autochtones et la justice. Rapport de la table ronde nationale sur les questions judiciaires, Commission royale sur les peuples autochtones. Ottawa: Ministère des Approvisionnements et Services.

Canada (2006). La justice en soi: traditions juridiques autochtones. Ottawa: Commission du droit du Canada.

Clastres, P. (1974). La société contre l'État. Recherches d'antbropologie politique. Paris: Éditions de Minuit.

Commission du droit du Canada (2003). Qúest-ce qu'un crime? Des défis et des choix. Document de discussion. Ottawa: Commission du droit du Canada.

Courtois, G. (1984). La vengeance, du désir aux institutions. In R. Verdier \& J.-P. Poly (Ed.), La vengeance. Études d'ethnologie, d'histoire et de philosophie, t. 3 : Vengeance, pouvoirs et idéologies dans quelques civilisations de l'Antiquité (7-45). Paris: Cujas.

Cousineau, M.-M., \& Cucumel, G. (1991). De la police au tribunal: formulation et cheminement des plaintes portées devant la Cour du Québec, chambre criminelle et pénale. Criminologie, 24 (2), 57-80.

Delâge, D., \& Gilbert, E. (2003). Les Amérindiens face à la justice coloniale française dans le gouvernement de Québec, 1663-1759. I: Les crimes capitaux et leurs châtiments. Recherches amérindiennes au Québec, 33 (3), 79-90.

Dorais, L.-J. (1984). Humiliation et harmonie. L'expression du droit coutumier chez les Inuits du Labrador. Recherches amérindiennes au Québec, 14 (4), 3-8.

Dorais, L.-J. (2009). Et si les autochtones n'existaient pas? In N. Gagné, T. Martin \& M. Salaün (Eds.), Autochtonies vues de France et du Québec (419430). Québec: Presses de l’Université Laval / Dialog.

Dumont, J. (1993). La justice et les peuples autochtones. In Canada, Les peuples autochtones et la justice. Rapport de la table ronde nationale sur les questions judiciaires (42-85). Commission royale sur les peuples autochtones. Ottawa: Ministère des Approvisionnements et Services.

Foucault, M. (1975). Surveiller et punir. Naissance de la prison. Paris: Gallimard.

Garfinkel, H. (1997). Practical Sociological Reasoning. Some Features in the Work of the Los Angeles Suicide Prevention Center. In M. Travers, J. F., Manzo (Ed.), Law in Action (25-42). Alderschot: Ashgate.

Garnot, B. (2000). Justice et société en France aux XVI $I^{e}$ XVII et XVIII siècles. Gap: Ophrys.

Hamilton, A., \& Sinclair, C. (1991). The Justice System and Aboriginal People: Report of the Aboriginal Justice Inquiry of Manitoba, 1, Winnipeg: The Queen's Printer.

Hénaff, M. (2000). La dette de sang et l'exercice de justice. In P. Dumouchel (Ed.), Violences, victimes et vengeances (31-64). Paris / Québec: L'Harmattan / Presses de l'Université Laval.

Honneth, A. (2000). La lutte pour la reconnaissance. Paris: Cerf.

Hulsman, L., \& Bernat de Celis, J. (1982). Peines perdues: Le système pénal en question. Paris: Le centurion. 
Jaccoud, M. (1995). Justice blanche au Nunavik. Montréal: Méridien.

Jaccoud, M. (1999). Les cercles de guérison et les cercles de sentence autochtones au Canada. Criminologie, 32 (1), 79-105.

Jaccoud, M., \& Johnson, L. (2007). Gouvernance autochtone et justice pénale. In A. Lajoie (Ed.), Gouvernance autocbtone: aspects juridiques, économiques et sociaux (204-213). Montréal: Thémis.

Javeau, C. (1994). Leçons de sociologie. Paris: Méridiens-Klincksieck.

Junger-Tas, J., Haen Marshall, I., \& Ribeaud, D. (Eds.) (2003). Delinquency in an International Perspective. The International Self-Reported Delinquency Study (ISRD). Amsterdam: Kugler.

Kellerhals, J., Coenen-Huther, J., \& Modak, M. (1988). Figures de l'équité. La construction des normes de justice dans les groupes. Paris: Presses universitaires de France.

Kellerhals, J., Modak, M., \& Perrenoud, D. (1997). Le sentiment de justice dans les relations sociales. Paris: Presses universitaires de France.

Lacasse, J.-P. (2004). Les Innus et le territoire. Innu tipenitamun. Québec: Septentrion.

Lajoie, A. (2008). Conceptions autochtones des droits ancestraux au Québec. Paris: Thémis.

Lalonde, L. (2003). La médiation pénale et la justice réparatrice: à toutes fins que de droit? In M. Jaccoud. (Ed.), Justice réparatrice et médiation pénale. Convergences ou divergences? (97-118). Paris: L'Harmattan.

Le Roy, E. (1999). La culture du pluralisme juridique est-elle soluble dans la criminologie? In J.-M. Bessette (Ed.), Crimes et cultures (61-80). Paris: L'Harmattan.

Lévesque, C. (2009). La recherche québécoise relative aux peuples autochtones à l'heure de la société du savoir et de la mobilisation des connaissances? In N. Gagné, T. Martin \& M. Salaün (Eds.), Autochtonies vues de France et du Québec (455-470). Québec: Presses de l'Université Laval / Dialog.

Llewellyn, K., \& Hoebel, E. (1999). La voie cheyenne. Conflit et jurisprudence dans la science primitive du droit. Paris: Librairie Générale de Droit et de Jurisprudence-Bruylant.

Martin, T. (2009). Pour une sociologie de l'autochtonisme. In N. Gagné, T. Martin, \& M. Salaün (Eds.), Autochtonies vues de France et du Québec (431454). Québec: Presses de l'Université Laval / Dialog.

Monture-OKanee, P. (1993). Rétablir la justice: les femmes autochtones et les initiatives en matière de justice dans les années 90 . In Canada, Les peuples autochtones et la justice. Rapport de la table ronde nationale sur les questions judiciaires (105-133). Commission royale sur les peuples autochtones. Ottawa: Ministère des Approvisionnements et Services.

Noreau, P. (2003). L'institutionnalisation de la justice réparatrice. In M. Jaccoud. (Ed.), Justice réparatrice et médiation pénale. Convergences ou divergences? (209225). Paris: L'Harmattan.

Paradelle, M. (2001). Du déterminisme du milieu. La notion de culpabilité dans la tradition juridique inuit, Actes des $\mathrm{XX}^{\mathrm{e}}$ Journées d'Histoire du Droit 
consacrées à la culpabilité, Cabiers de l'Institut d'Antbropologie juridique, 6, 127-158.

Pires, A. (2001). La rationalité pénale moderne, la société du risque et la juridicisation de l'opinion publique, Sociologie et sociétés, 32 (1), 179-204.

Pollner, M. (1991). «Que s'est-il vraiment passé?». Événement et monde commun. In J.-L. Petit (Ed.), L'événement en perspective (75-96). Paris: École des hautes études en sciences sociales.

Robert, P. (1974). Sociologie criminelle: la sociologie entre une criminologie du passage à l'acte et une criminologie de la réaction sociale. L'Année sociologique 1973. Paris: Presses universitaires de France.

Robert P. (1977). Les statistiques criminelles et la recherche, réflexions conceptuelles. Déviance et société, 1 (1), 3-27.

Ross, R. (1996). Returning to the Teachings. Exploring Aboriginal Justice. Toronto: Penguin.

Rouland, N. (1983). L'acculturation judiciaire chez les Inuit du Canada. Recherches Amérindiennes au Québec, 13, $1^{\text {re }}$ partie $n^{\circ} 3,179-191,2^{\mathrm{e}}$ partie $\mathrm{n}^{\circ} 4$, 307-318.

Simard, J.-J. (2003). La réduction. L'autochtone inventé et les Amérindiens d'aujourd'bui. Québec: Septentrion.

Simon, S. (2009). Identité autochtone et lutte pour l'autodétermination: le cas de la nation taroko à Formose. In N. Gagné, T. Martin \& M. Salaün (Eds.), Autochtonies vues de France et du Québec (311-330). Québec: Presses de l'Université Laval / Dialog.

Sioui, G. (1999). Pour une histoire amérindienne de l’Amérique. Québec: Presses de l'Université Laval.

Strimelle, V. (2007). La justice réparatrice: une innovation du pénal? Champ Pénal/Penal Field, 4, http: www//champpenal.revues.org/documents912.html.

Swidler, A. (1986). Culture in Action: Symbols and Strategies. American Sociological Review, 51 (2): 273-286.

Verdier, R. (1980). Le système vindicatoire. Esquisse théorique. In R.Verdier, J.-P. Poly \& B. Courtois (Eds.), La vengeance. Études d'ethnologie, d'bistoire et de philosophie, t.1: Vengeance et pouvoir dans quelques sociétés extra-occidentales (11-42). Paris: Cujas.

Verdier, R. (1984). Une justice sans passion, une justice sans bourreau. In Verdier, R., J.-P. Poly (ed.), La vengeance. Études d'ethnologie, d'bistoire et de philosophie, t.3: Vengeance, pouvoirs et idéologies dans quelques civilisations de l'Antiquité (149-153). Paris: Cujas.

Verdier, R. (2004). Histoire du monopole étatique de la vengeance en Occident, In R. Verdier (Ed.), Vengeance. Le face-à-face victime/agresseur (145-159). Paris: Autrement.

Watzlawick, P., Weakland, J., \& Fisch, R. (1975). Changements. Paradoxes et psychothérapie. Paris: Seuil. 
ABSTRACT - Our research focuses on the following questions: what is a disorder, a conflict? How do we define them and react to them? Our main hypothesis is based on the existence of an active justice model which regulates our social exchanges but is hidden by the dominant discourse of the penal justice: the "vindicatory" model. In this article, we shall more particularly discuss issues raised by the meeting between penal and vindicatory models, and describe how these two regulation models could mix. In that purpose, we shall focus on the regulation modes used by the Aboriginal peoples living in Quebec. We shall first describe how they define and handle the different forms of confusion which emerge in their communities. By doing this, we shall then try to identify the vindicatory aspects of their regulation practices and discuss how these models survived when confronted to the western legal and penal model.

KEYWORDS - Aboriginal Peoples, Troubles and Conflicts Regulation, Vindicatory, Penal, Legal Pluralism.

RESUMEN • ¿Qué es un trastorno, qué un conflicto? ¿Cómo los definimos, cómo reaccionamos ante ellos? Estas preguntas, trama básica del presente artículo, forman parte de los trabajos actuales que exploran la hipótesis de la existencia de un modelo de justicia activo en nuestras relaciones sociales pero que se esconde en el discurso dominante de la justicia penal: el modelo vindicativo. Analizaremos, en particular, las cuestiones planteadas por el encuentro entre los dos modelos de regulación, el penal y el vindicativo y la forma en que ambos modelos se tocan. Para ello, abordaremos los modos de regulación de problemas entre la población autóctona de Quebec. Luego de analizar su definición de trastorno y las formas de regularlo, trataremos de mostrar la cara vindicatoria de este sistema para en seguida describir cómo los modelos autóctonos de regulación y el sistema de justicia penal se tocan. Planteamos, finalmente, diversos aspectos de reflexión respecto de la sobrevivencia y adaptación de dicho modelo en el contexto jurídico-penal occidental.

PALABRAS CLAVE - Autóctonos, regulación de trastornos y conflictos, vindicativo, penal, pluralismo jurídico. 